\title{
Propuesta metodológica docente y fabricación digital de una pieza de mobiliario urbano: de la idea a la realidad en 10 horas
}

\author{
Sánchez Gómez, Santiago ${ }^{\mathrm{a}}$; Bono Cremades, Javier ${ }^{\mathrm{b}}$; Ramón Constantí, Amanda ${ }^{\mathrm{c}}$; \\ Martínez Usó, Jose ${ }^{\mathrm{d}}$ \\ ${ }^{a}$ Arquitecto. Universidad Politécnica de Valencia, España, santi.sg.arq@gmail.com ${ }^{\mathrm{b}}$ Arquitecto. \\ Universidad Politécnica de Valencia, España, jbonocremades@gmail.com ${ }^{\mathrm{c}}$ Arquitecta. Universidad \\ Politécnica de Valencia, España, amracon@arq.upv.es, ${ }^{\text {A} A r q u i t e c t o . ~ U n i v e r s i d a d ~ P o l i t e ́ c n i c a ~ d e ~}$ \\ Valencia, España, jomarus641984@gmail.com
}

\begin{abstract}
Resumen
La forma de enfocar la docencia y los diferentes procesos educativos llevados a cabo en las Escuelas de Arquitectura de todo el mundo está cambiando. Existe una búsqueda cada vez más apresurada por lograr involucrar y sumergir a los alumnos en las dinámicas que, eventualmente, los preparará como futuros arquitectos y que, sin embargo, empiezan a verse influenciadas por procedimientos cada vez más rápidos, nuevas necesidades formativas o el uso de nuevas tecnologías, entre otros. ¿Es posible darle un giro a la docencia en una institución con unos currículums tan reglados y lograr, al mismo tiempo, complementarla a través de actividades como un festival de arquitectura?
\end{abstract}

En el marco de la cuarta edición consecutiva del Festival de Arquitectura de la Escuela Técnica Superior de Arquitectura de Valencia (ETSA.TOPIA), organizado por alumnos de los diferentes cursos de Grado y Máster contando con la implicación de profesores, personal de la Escuela y colaboradores externos se ha podido comprobar un año más cómo actividades de este tipo enriquecen no sólo la atmósfera cotidiana fuera de las aulas si no también, la actividad docente mediante el desarrollo de talleres o actividades que puedan contribuir en la adquisición de competencias trasversales, u ofrecer comprobación empírica de aquello que se estudia en clase de una forma amena y participativa.

Así, en la reciente celebración de ETSA.TOPIA y en relación con el tema propuesto para esta edición, se realizó un taller de introducción al diseño paramétrico y a la fabricación digital en colaboración con grupos adscritos al programa "Generación Espontánea" donde se logró construir una pieza de mobiliario urbano a partir de un modelo adaptativo empleando la técnica de impresión en hormigón. Se pretende analizar la experiencia realizada y llegar a unas conclusiones aplicables a futuras actividades o recursos docentes que contribuyan en el desarrollo de los profesionales surgidos de la Escuela de Arquitectura de Valencia. 
Propuesta metodológica docente y fabricación digital de una pieza de mobiliario urbano: de la idea a la realidad en 10 horas

Palabras clave: fabricación digital, festival de arquitectura, ETSA Valencia, docencia.

\section{Introducción: la docencia en arquitectura actualmente y hacia dónde se dirige}

La formación del estudiante de arquitectura en las escuelas españolas ha seguido históricamente un aprendizaje creativo fundamentado de forma técnica (Domingo Santos, 2010), respecto a otras instituciones docentes europeas. Se espera del alumno desarrollar una serie de competencias (como la comunicación efectiva, el pensamiento crítico, planificación y gestión del tiempo, entre otras) a través del trabajo prueba-error desarrollado en el aula. No obstante, podemos decir que la formación de los estudiantes de arquitectura de todo el mundo cuentan con unas características y siguen unas directrices más o menos similares, sin tener en cuenta las competencias que puedan asumir los técnicos y profesionales arquitectos dependiendo del lugar donde ejerzan.

En conclusión y de forma generalizada, la docencia en arquitectura se basa en la aplicación por parte del alumnado del pensamiento crítico, los procesos prueba-error, el trabajo en equipo, la visión espacial, el trabajo y aplicación de modelos a escala, entre otros. El conocimiento de diferentes procesos de construcción, cálculo y modelización, así como el estudio de la historia y cultura constituyen de una forma más acotada el ámbito delimitado por el Plan Bolonia que actualmente estructura la enseñanza de los Grados y Másteres específicos en arquitectura que podemos encontrar en las universidades españolas.

\section{La fabricación digital como herramienta de diseño y construcción arquitectónicas}

A día de hoy se ha producido un nuevo planteamiento en los métodos constructivos, tanto a nivel conceptual como pragmático, gracias a la aparición de la fabricación digital. Ésta comprende una serie de procesos realizados mediante software de diseño asistido por ordenador tipo CAD (Kardos \& Váncza, 2018) los cuales posteriormente, se sistematizan y traslada su producción y manufactura utilizando maquinaria especializada (J. Seely, 2004), que puede comprender tecnología como cortadoras láser y $\mathrm{CNC}$, impresoras $3 \mathrm{D}$, etcétera. Estos procesos informatizados comenzaron a aplicarse a finales de la década de 1980, sistematizando procesos y geometrías complejas (Tapia Abril, 2014). 
Así, la fabricación digital como proceso y herramienta de diseño aplicados a la arquitectura, tanto en el aspecto generador de geometría y volumetría, la optimización de éstas para lograr un mejor rendimiento o economización de los materiales, o el aspecto más técnico aplicado a la fabricación de elementos o piezas específicas, comprende una serie de técnicas que a pesar de llevar aplicándose desde hace casi dos décadas, son aún hoy relativamente novedosas.

\subsection{Medios y recursos requeridos en procesos que comprendan el uso de fabricación digital}

El desarrollo de cualquier actividad o ejercicio práctico relacionado con el aprendizaje de arquitectura requiere de espacios y herramientas de trabajo concretos mediante las cuales, los alumnos puedan desarrollar modelos a escala y otro tipo de material tangible. Esto implica no sólo una evidente inversión económica, sino también, una asignación de un espacio concreto y una organización que regule el uso de la maquinaria y recursos disponibles entre los alumnos, lo que afectará directamente a la manera de organizar los grupos docentes y mantener una proporción y ratios adecuadas entre alumnos-profesor. Como ejemplo, escuelas de arquitectura como la de la ETH de Zurich o el Massachusetts Institute of Technology cuentan con programas de máster y postgrados especializados en esta materia, que permiten a la propia institución académica contar con unos recursos y atención especializada y que actualmente, si hacemos referencia a una gran mayoría de escuelas de arquitectura españolas, sólo es posible lograr junto a la colaboración de los llamados FabLabs (International Conference on Education and New Learning Technologies (9 : 2017 : Barcelona), Gómez Chova, López Martínez, \& Candel Torres, 2017).

Esta organización de grupos, gestión del espacio y recursos disponibles, así como el marco docente dentro de la Universidad Politécnica de Valencia, se tratarán posteriormente en esta comunicación utilizando como ejemplo una experiencia llevada a cabo en la Escuela Técnica Superior de Arquitectura de Valencia, tratando de extraer unas metodologías docentes básicas en este ámbito, cuya aplicación podría resultar interesante plasmar en futuras experiencias similares. 


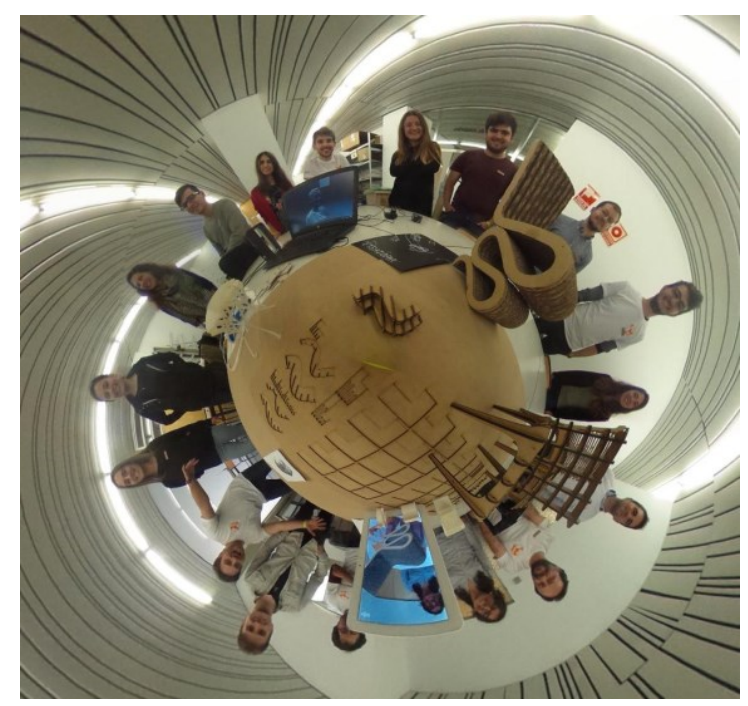

Fig. 1 Fotografia 360 del taller "Construye lo imposible”. Elaboración propia.

\section{El caso de la Escuela Técnica Superior de Arquitectura de Valencia}

Actualmente, la mayoría de alumnos matriculados en la Escuela Técnica Superior de Arquitectura de Valencia se encuentran cursando el denominado "Grado en Fundamentos de la Arquitectura", con 360 créditos repartidos en 5 cursos según el plan Bolonia. Estos 5 cursos aúnan los conocimientos y competencias básicas que se esperan de un arquitecto, siendo el Máster en Arquitectura posterior (habilitante) el recorrido académico mayoritario escogido por los alumnos una vez finalizado dicho Grado.

Tanto el Grado como el Máster anteriormente citados cuentan con asignaturas obligatorias y optativas que contribuyen en la formación de los alumnos en aspectos como la visión espacial, la manipulación y construcción de modelos a escala, así como el pensamiento crítico y trabajo del detalle arquitectónico. Esto se evidencia en materias como Dibujo Arquitectónico, Geometría Descriptiva, Proyectos o Construcción donde se orienta al alumno en cuanto a la utilización de diferentes softwares como herramienta para lograr el objeto arquitectónico buscado. Además, se alienta la comprensión del espacio mediante la utilización de maquetas realizadas con técnicas y materiales que pueden, o no, incluir metodologías de fabricación digital, si bien éstas no son objeto específico de ninguna materia per se. Todavía no existe ninguna asignatura específica en la que se traten procedimientos concretos de fabricación digital, tanto para la realización de modelos a escala, como aplicados al diseño de edificios o cualquiera de sus elementos o incluso, aplicada a la optimización de elementos como podrían ser fachadas o incluso estructuras. 


\subsection{El festival de cultura y arquitectura ETSA.TOPIA: cronología y organización}

ETSA.TOPIA es la respuesta en forma de festival de cultura y arquitectura promovido por los alumnos de la Escuela de Arquitectura de Valencia a incógnitas como qué futuro espera a los jóvenes arquitectos egresados, de qué estará hecha la arquitectura dentro de pocos años o cómo podemos abrazar la cultura y técnicas de construcción locales para lograr propuestas arquitectónicas globales. Desde el año 2015, la Escuela de Arquitectura de Valencia acoge este festival que, con la colaboración de alumnos, profesores, y personal no docente transforma durante su celebración la Escuela para realizar talleres, charlas, conferencias y actividades de diversa índole siempre con un trasfondo didácticoarquitectónico (Equipo de Festival Etsa.Topia, 2017). Algunos de estos talleres sirven como banco de pruebas donde experimentar metodologías docentes innovadoras en el marco de la Escuela, pero de una manera que no comprometa el currículum académico descrito anteriormente, actualmente muy reglado en cuanto a horas de dedicación y contenido.

\section{El taller "Construye lo imposible": organización, metodología, didáctica y objetivos}

En el marco de la cuarta edición del festival ETSA.TOPIA, cuya temática fue "360" y celebrada entre el 19 y el 21 de abril de 2018 se propuso el taller "Construye lo imposible" mentorizado por el grupo "I+D+Arq"1 y en colaboración con el equipo de "BeMore3D" 2 . El taller, que tuvo una duración de un día entero (de nueve y media de la mañana a ocho de la tarde, con una pausa a mediodía) estaba dividido en dos bloques: teórico y práctico, y donde intervinieron ambos grupos como mentores del grupo de alumnos que acudió al aula esa jornada. En total, se reunieron cinco alumnos y un profesor de la asignatura Proyectos Arquitectónicos, que actuaban como mentores en las diferentes fases del taller propuesto, al cual asistió un grupo de trece alumnos, inscritos previamente. Este workshop consistía en una introducción a la fabricación digital aplicada al diseño de arquitectura, así como diferentes métodos de visualización y realidad virtual (cada vez más extendidos en el campo del patrimonio arquitectónico y cultural) para posteriormente, trabajar in-situ con la

\footnotetext{
1 I+D+Arq es un grupo adscrito al programa "Generación Espontánea” de la Universidad Politécnica de Valencia, con interés por la investigación e innovación aplicadas al diseño, la proyectación arquitectónica y el urbanismo.

2 BeMore3D es una empresa fundada en Valencia en 2015, especializada en la realización de prototipos en plástico y otros materiales utilizando tecnologías de impresión 3D. Actualmente, centran su labor en la construcción de viviendas económicas mediante esta tecnología, aplicada mediante una máquina impresora 3D gran escala para su uso con materiales cementicios.
} 
tecnología de impresión 3D en hormigón desarrollada por "BeMore3D". La proporción entre alumnos y mentores ya resulta significativa, y fue previamente acordada por éstos últimos, teniendo en cuenta que una clase magistral de Proyectación Arquitectónica puede albergar grupos de entre cuarenta y cincuenta alumnos atendidos por dos profesores, y donde resultaría imposible para un único docente dedicar el tiempo y atención a todos los estudiantes, si existiese una asignatura de características similares a este workshop en el currículum reglado. Tampoco sería posible dedicar los recursos actualmente disponibles en la Escuela a tal cantidad de alumnos de forma simultánea.

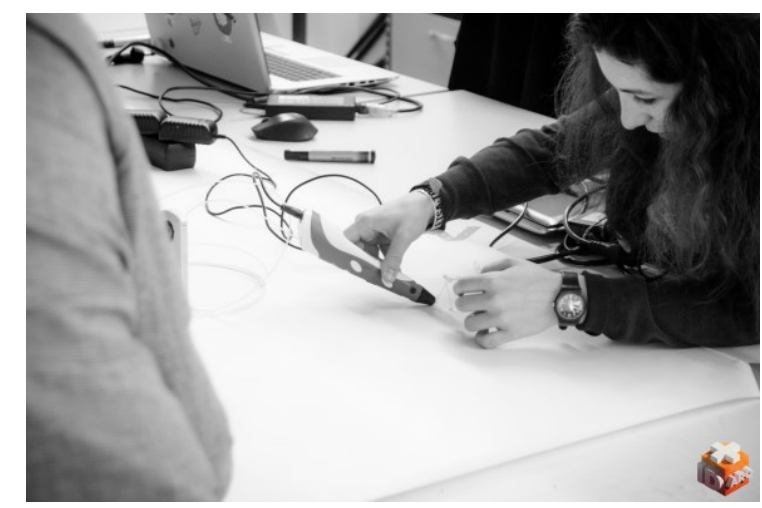

Fig. 2 Alumnos del taller descubriendo nuevas formas de proyectar. Elaboración propia.

El objetivo del taller era generar junto a los alumnos una pieza de mobiliario urbano creada mediante un software de diseño paramétrico (Rhinoceros + plugin Grasshopper) para posteriormente, manipularlo con una aplicación online gratuita y variar algunas de sus características. Con esto se pretendía demostrar también la utilidad en la arquitectura y el diseño de la posibilidad de ofrecer al usuario o cliente final la posibilidad de crear piezas mediante diseño adaptativo. Los alumnos inscritos pertenecían a diferentes niveles académicos dentro del Grado en Arquitectura, y a pesar de que todos ellos contaban con un conocimiento muy básico de los programas de modelado utilizados, ninguno de ellos había trabajado previamente con tecnología que permitiese trasladar un modelo de este tipo a la realidad mediante la tecnología mencionada. No obstante, no surgieron dudas significativas después de la exposición del ejercicio ni de las pautas establecidas a la hora de afrontar el prototipo, si no que la utilización del pensamiento crítico, analítico y la relación con conocimientos previos (Prusak, 2004) permitieron desarrollar la actividad sin altibajos ni pausas significativas. De hecho, el tamaño del grupo, considerado mediano, permitió establecer un debate muy interesante acerca de la forma y función que debía tener un banco de exterior de las características que se requerían y con un enfoque constructivo tan específico como era el de trasladarlo posteriormente a una máquina impresora de materiales cementicios. 
Este hecho quizá no habría sido posible de haber contado con un grupo mucho más reducido o, tal y como se comentaba anteriormente, un grupo de características similares a las que se encuentran en cualquier aula en las horas destinadas a Proyectos Arquitectónicos, con una ratio alumno-profesor mucho mayor. Resultó patente, además, que el nivel académico en que se encontraban los alumnos no era significativo, ya que todos ellos aportaron ideas y contribuyeron al desarrollo de la clase de manera activa.

Uno de los problemas más significativos con los que nos encontramos a la hora de organizar el workshop fue el tema relacionado con la logística. A pesar de que la impresora 3D de gran formato estaba previamente instalada y preparada para su utilización en el espacio cedido para ello en el campus, se utilizaron como introducción a las diferentes tecnologías tratadas una serie de dispositivos adicionales, como un escáner 3D (Chen, 2008), lentes de realidad aumentada y aparatos similares, así como la totalidad de ordenadores portátiles de los alumnos conectados a la red eléctrica del aula para poder seguir el desarrollo del taller. La falta de enchufes en el aula evidenció la necesidad de que éstas estén adaptadas para un posible futuro docente en el que la utilización continua de estos dispositivos forme parte del día a día en una Escuela de Arquitectura. Además, a pesar de contar con el permiso necesario para acceder y utilizar las impresoras 3D con las que cuenta la Escuela en su taller de maquetas, la distancia a éste respecto al aula ocupada para la actividad, y el aforo máximo que permite la sala donde se encuentran dichas máquinas, hacían inviable su utilización en el workshop con objetivo de realizar tests del prototipo antes de pasar a la gran escala.

El resultado de la primera parte del taller se plasmó en un banco corrido de forma ondulante, sin respaldo, de una altura aproximada de 45 centímetros y anchura de 2 metros, que se trasladó a la maquinaria ubicada en el exterior, donde alumnos, mentores y técnicos contribuyeron a su puesta en marcha, preparación y disposición del material en la máquina, así como la supervisión del proceso de impresión. A pesar de que aproximadamente la mitad de los alumnos del taller no contaban con experiencia previa en un entorno real de puesta en obra, y teniendo en cuenta los contratiempos que podrían considerarse típicos de una experiencia como esta (ajustes de última hora en el diseño, el ajuste y corte de las varillas de armado intermedio en las tongadas de hormigón, o posibles obstrucciones en la máquina impresora, entre otros), la colaboración y consejos de los técnicos y mentores permitieron llevar a cabo la experiencia de una forma muy satisfactoria, con unos alumnos que al terminar la jornada, manifestaron su interés por aprender más sobre el tema de la fabricación digital y su posible aplicación práctica en el entorno del Grado en Arquitectura y sus futuros proyectos académicos y/o profesionales. 


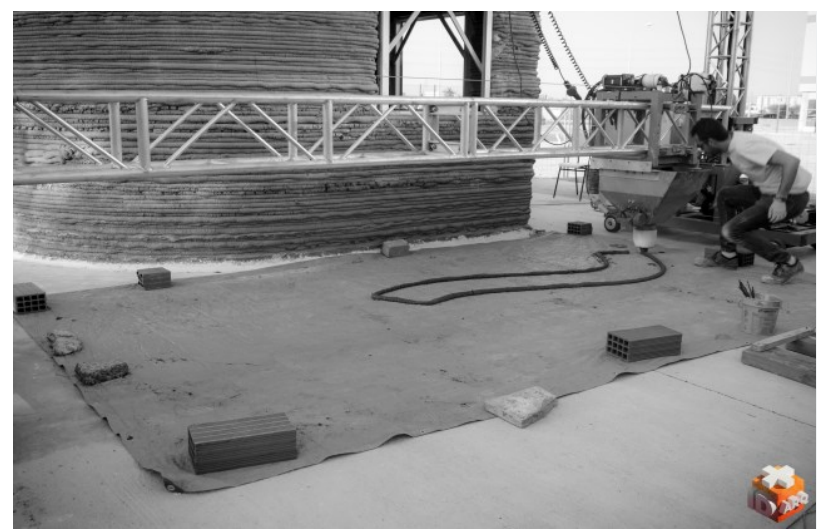

Fig. 3 Impresora 3D de hormigón de la empresa "BeMore3D” comenzando la impresión del banco corrido

\section{Conclusiones obtenidas a raíz del taller. Metodologías en la introducción de la fabricación digital a la docencia arquitectónica}

Tras la realización de la actividad en el taller, vimos un marcado interés tanto del alumnado como de los propios organizadores del evento, por la subyacente investigación y la generación de sinergias multidisciplinares que se transmitían en cada una de las actividades desarrolladas. Las reflexiones se centraron en:

- Nuevas tecnologías y metodologías de aplicación física

- Uso concreto en modelos paramétricos basados en arquitectura procedural

- Investigación en software paramétrico open-source y libre

- Exportación a modelos físicos de forma automatizada

- Prototipado rápido de producto

Quedó patente el hecho de que los planes de estudio oficiales carecen de una constante actualización tanto en metodología proyectual, como conocimiento de las últimas versiones del software que las permiten, con lo que se ven abocados a un desfase generacional continuado que se aleja de las necesidades reales del mercado y la sociedad fuera de los ámbitos puramente académicos.

Un hecho a destacar fue, que gran parte de los organizadores del evento somos profesionales autónomos o contratados, que ejercemos una tarea profesional externa a la universidad, lo que nos permite (y hasta cierto punto obliga), a estar constantemente actualizados en conocimiento y manejo de software para así poder tener una posición competitiva en el mercado. Este hecho que puede parecer anecdótico, es la clave para una mejora sustancial en el modelo educativo actual en la docencia de arquitectura, ya que la revolución industrial continuada en la que vivimos requiere de una actualización constante, ya no solo a nivel práctico sino incluso conceptual. Este hecho queda 
patente en el desarrollo de la segunda fase del taller, en el que el alumnado exportó y fabricó mediante impresión $3 \mathrm{D}$ en hormigón mobiliario urbano real en un muy corto periodo de tiempo.

Además, en la realización de este taller resultó evidente la falta de herramientas en el aula destinadas a aplicar estas nuevas metodologías docentes, basadas en la utilización de recursos y herramientas destinadas a que el alumno realice sus prototipos y maquetas. Herramientas actualmente presentes en la infraestructura de la Escuela, pero con carencias importantes a la hora de llevar esta aplicación a la práctica, ya que un alumno promedio desconoce cómo modelar y exportar sus proyectos para ser trasladados a una de las impresoras 3D disponibles en la Escuela. Incluso, la tecnología utilizada para el corte láser y la construcción de maquetas mediante esta herramienta presente en la Escuela de Arquitectura de Valencia, es aún hoy un hecho que los alumnos aprenden a aplicar casi en los últimos cursos del Grado, y normalmente, gracias a compañeros que han hecho uso de tal maquinaria previamente, a pesar de la existencia de una página web donde encontrar las instrucciones y directrices de uso. Esto prueba que quizá resultaría de interés incluir de alguna manera en el plan de estudios actual, no una asignatura a tiempo completo, pero sí algún módulo o tema enfocado a enseñar a los alumnos unas pautas básicas para trabajar con estas herramientas actualmente disponibles.

Aún así, consideramos que el taller fue un éxito no solo por la construcción final de la pieza, sino por la generación de una línea conceptual-productiva basada en una renovación crítica continuada de los medios de producción, tanto a nivel de ideación paramétrica, generación de prototipos automatizados, actualización y personalización de dichos prototipos con un elevado control procedural y la aplicación de la fabricación digital como nuevo paradigma metodológico constructivo.

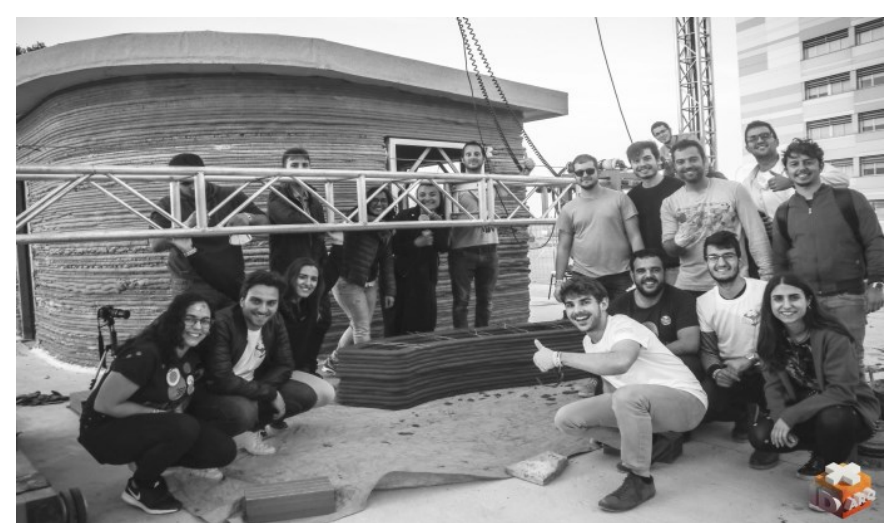

Fig. 4 Alumnos, mentores y técnicos participantes en el taller, con el modelo final construido. Elaboración propia. 
Propuesta metodológica docente y fabricación digital de una pieza de mobiliario urbano: de la idea a la realidad en 10 horas

\section{Referencias}

Chen, T. (2008). New 3D Scanning Techniques for Complex Scenes. Saarbrücken, Germany. Retrieved from https://diglib.eg.org/xmlui/bitstream/handle/10.2312/8201/chen.pdf?sequence=1

Domingo Santos, J. (2010). Nuevas ilusiones para una escuela. Revista Proyecto, Progreso, Arquitectura, $N^{\circ} 1,92-99$. Retrieved from https://revistascientificas.us.es/index.php/ppa/article/view/199/197

Equipo de Festival Etsa.Topia. (2017). ETSA·TOPIA 2017. Retrieved September 24, 2018, from https://sway.office.com/W7ZOETh3zA2GnYAq

International Conference on Education and New Learning Technologies $(9: 2017$ :

Barcelona), A., Gómez Chova, L., López Martínez, A., \& Candel Torres, I. (2017).

Edulearn 17 conference proceedings. EDULEARN17 Proceedings. IATED Academy. Retrieved from https://library.iated.org/view/MARTINPASTOR2017DIG

J. Seely. (2004). Digital fabrication in the architectural design process, (1999), 1-121.

Kardos, C., \& Váncza, J. (2018). Application of Generic CAD Models for Supporting Feature Based Assembly Process Planning. Procedia CIRP, 67, 446-451. https://doi.org/10.1016/j.procir.2017.12.240

Prusak, Z. (2004). Mistake-Proofing as the Base for Teaching Principles of Engineering Problem Solving. In 2004 Annual Conference (p. 10). Central Connecticut State University.

Tapia Abril, V. E. (2014). Contemporary design and digital fabrication. Estoa, 3(4), 39-43. https://doi.org/10.18537/est.v003.n004.05 